\title{
Shall I commence the breathing, admiral? Zur Ikonographie von Atmung und Atemnot im Science Fiction-Film
}

\begin{abstract}
Kein Umfeld ist für Wesen, deren Organismus sich evolutionär unter den physikalischen Bedingungen des Planeten Erde entwickelt hat, lebensfeindlicher als das Weltall. Dieses Faktum wird nicht nur zur technischen Herausforderung in der realen bemannten Raumfahrt, sondern ebenso zum zentralen Motiv in den audio-visuellen und narrativen Welten des Kinos als der wohl wirkungsmächtigsten Bilder- und Geschichtenmaschine der Moderne. Im Science Fiction-Film überlagert der existentielle Vorgang des Atmens dramaturgisch sämtliche weiteren Aspekte wie etwa fehlende Gravitation oder Strahlungsbelastung.
\end{abstract}

\section{Darth Vaders Mienenspiel, das Loch im Helm}

In seiner Kombination aus schwarzer Uniform, mit wehendem Mantel sowie Samurai-reminiszentem Helm, und dem bedrohlichen Röcheln der Respiration gehört der audio-visuelle Auftritt des Erzschurken Darth Vader in der Star Wars-Saga unzweifelhaft zu den eindrücklichsten Ikonen der kinematographischen Raumfahrt. Der auditive Aspekt von Darth Vaders Atmung wurde Mitte der 1970er Jahre von Sound Designer Ben Burtt aus modifizierten Aufnahmen eines Drucklufttauchgeräts entwickelt und ist als Soundeffekt patentgeschützt. ${ }^{1}$ Das berühmteste Atemgeräusch der Science Fiction-Filmgeschichte findet sich nicht nur auf Youtube in bis zu zehnstündigen Loop-Versionen, ${ }^{2}$ sondern inspirierte auch zahllose Parodien. Der Helm verunklärt sowohl Mienenspiel als auch sprachliche Artikulation des dunklen Sith Lords. Eine Parodie aus der Peter Serafinowicz-Show der BBC zieht

1 Vgl. zum Sound-Design von Star Wars allgemein Jonathan

W. Rinzler, The Sounds of Star Wars, London 2010.

2 "10 Hours of Darth Vader breathing“, Video, 10:00:00, verfügbar unter: https://www.youtube.com/watch?v=9VBm5xNobrs (letzter Zugriff: 5.6.2020). 
wortspielerischen Witz aus dieser unheimlich-undeutlichen Aussprache, wenn in der Lagebesprechung aus der Frage „Shall I commence the briefing, admiral?" mit Lord Vaders Trade Mark-Atmung ein unverwechselbar geröcheltes „Shall I commence the breathing?" wird. ${ }^{3}$

Auch in der realen Raumfahrt beeinträchtigen Raumanzüge die Gestik und Mimik ihrer Träger und Trägerinnen, wie vielleicht die Bilder der ersten Mondlandung 1969 für die Weltöffentlichkeit am nachdrücklichsten veranschaulichen. Buzz Aldrins Gesicht bleibt unsichtbar; sein spiegelndes Helmvisier reflektiert lediglich den mit ihm über den Mond tapsenden Neil Armstrong.

In der kinematographischen Raumfahrt gerät der Raumanzug zum dramaturgischen Verhandlungsort zwischen Wahrscheinlichkeitsanspruch und ästhetischer Eigengesetzlichkeit des Films. In einer Standardszene wird kurz vor der Landung auf dem fremden Planeten die Atmosphäre der extraterrestrischen Welt analysiert, wobei sich mit planetologisch erstaunlicher Häufigkeit herausstellt, dass ihr Gasgemisch für den Menschen atembar, ein Helm mit Sauerstoffversorgung daher nicht vonnöten ist - und Mimik und Gestik sich ungehindert entfalten können. Die filmhistorische Tradition lässt sich abstecken von Georges Méliès' Voyage dans la Lune (Frankreich 1902), der die langbärtigen irdischen Besucher in gutbürgerlicher Garderobe und Zylinder auf den Erdtrabanten führt, über Gerda Maurus als Frau im Mond (Deutschland 1929), die zwar schweres Schuhwerk gegen die geringere Schwerkraft gebraucht, ansonsten jedoch auf zeitgenössische Theorien zu Sauerstoffvorkommen auf dem Mond vertraut, und über das Episoden-Gros der originalen Star Trek-Serie (USA 1966-69) bis hin noch zu Interstellar (USA/Großbritannien 2014). Die prototypische und popkulturell verinnerlichte Situation aus Star Trek zeigt die Crew der Enterprise heruntergebeamt auf einen Planeten, dessen atmosphärische Verhältnisse die Schauspieler von darstellerisch hinderlichem Raumanzug respektive Helm befreien. Tim Burtons Mars Attacks! (USA 1996) wird diese Konzeption mit dem Martian Girl parodieren, dem sexy piece, das bei einer Aufklärungsstippvisite der fiesen Aliens dem Presseberater des Präsidenten im Weißen Haus den Finger abbeißt, als dieser versucht, dem vermeintlichen Lustobjekt den unerlässlichen Stickstoff-Atemkaugummi aus dem Mund zu klauben.

3 Vgl. „Darth Vader in Love“, Video, 00:06:48, TC 00:00:0000:00:25, verfügbar unter: https://www.youtube.com/ watch?v=0X9NFknjTRE (letzter Zugriff: 5.6.2020). 

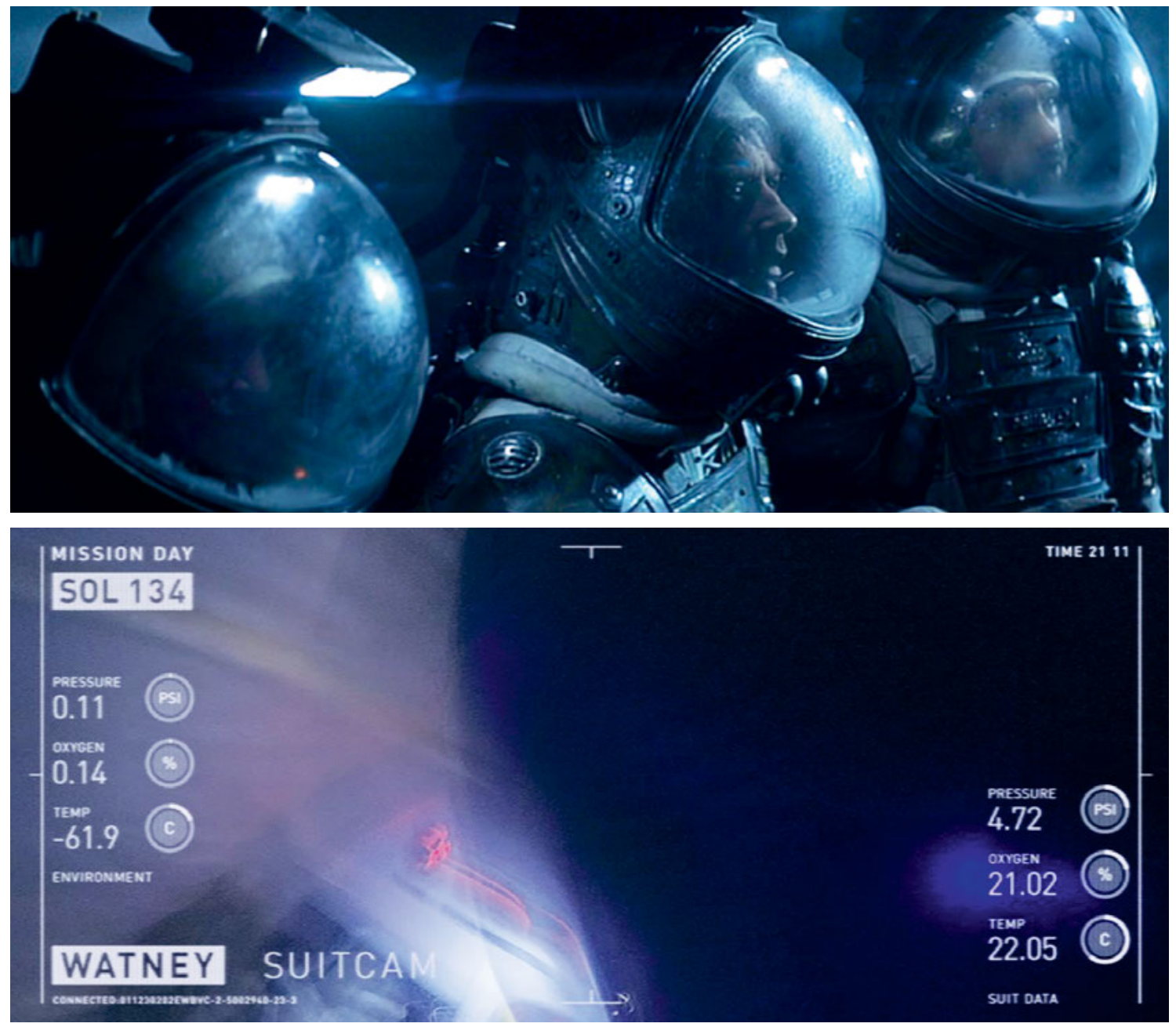

Abb. 2: Kreatürliches Chaos und objektive Messdaten, The Martian, 2015, Screenshot 
Der Verzicht auf aufwendige Raumanzüge diente bei Produktionen wie dem frühen Star Trek sicherlich auch der Kostenersparnis, doch das vestimentäre Problem der Schauspielerinnen und Schauspieler musste ebenso in sich realistischer gebenden Filmen bewältigt werden. So tragen etwa die drei Besatzungsmitglieder der Nostromo, die in Ridley Scotts Alien (Großbritannien/USA 1979) auf der Suche nach der Quelle eines aufgefangenen Signals auf LV-426 gelandet sind, Raumanzüge mit dem Fishbowl Helmet, den die NASA für die Apollo-Missionen entwickelt hatte und der ein weites Sichtfeld für den Astronauten ermöglicht. ${ }^{4}$ Man könnte meinen, die NASA hätte zugleich an die Bedürfnisse Hollywoods gedacht, ermöglicht der Fishbowl Helmet doch im Film auch den Blick von außen in das Innere des Helms und erlaubt der Kamera, bei geeigneter Ausleuchtung und Kameraführung, das Gesicht des Protagonisten durch das Helmvisier zu zeigen. In Alien hält John Hurt als Kane die verbale Kommunikation mit dem Mutterschiff aufrecht (Abb. 1): wir sehen ihn sprechen und seine mimischen Reaktionen. Die Gesichter seiner beiden Gefährten, nicht unmittelbar an diesem Gespräch beteiligt, sind hingegen undeutlicher oder gar nicht zu erkennen aufgrund der Lichtreflexe auf den Helmen oder auch des Kondensats der Atmung, das sich von innen am Visier niederschlägt. ${ }^{5}$

Die Luft, die wir atmen, ist unsichtbar, die Atmung im Normalfall nicht zu sehen, kaum zu hören. Auf der postapokalyptischen Venus im Schweigenden Stern (DDR/Polen 1960) ist die Atmosphäre giftig und radioaktiv verseucht. Szenenbildner Alfred Hirschmeier übersetzte unsichtbares Gasgemisch und unsichtbare Strahlung in ersten Scribbles und elaborierten Farbentwürfen sofort in horizontal wabernde Dunstschleier, die den Weg der irdischen Venus-Besucher suggestiv kreuzen. Im resultierenden Film werden realer Nebel und tricktechnische Schlieren in Rot und Gelb die Filmbilder durchziehen und die lebensfeindliche Zusammensetzung der Venus-Atmosphäre in gefährlich leuchtenden Warnfarben visualisieren. ${ }^{6}$

Das Kondensat der Atemluft auf der Innenseite des Visiers beim Alien-Expeditionstrupp und die gespenstische Szenerie im Schweigenden Stern veranschaulichen, dass es im Science Fiction-Film vor allem Gefahrensituationen sind, auf die sich die bewegt audio-visuellen

4 Zur Entwicklung der US-Raumanzüge siehe Kenneth S. Thomas/Harold J. McMann, US Spacesuits, Berlin u.a. 2006; informativ zur popkulturellen Verwendung des Motivs der Eintrag zum Fishbowl Helmet unter https:// tvtropes.org/pmwiki/pmwiki.php/Main/FishbowlHelmet (letzter Zugriff: 5.6.2020).

5 Vgl. Ridley Scott, Alien, DVD, Twentieth Century Fox Home Entertainment 2012, um TC 00:20:36.
6 Vgl. Marcus Becker, „Die Sache mit dem Weltniveau. Die DEFA-Science-Fiction und das Szenenbild eines Filmgenres“, in: Annette Dorgerloh/Marcus Becker (Hg.), Alles nur Kulisse?! Filmräume aus der Traumfabrik Babelsberg, Weimar 2015, S. 158-167, hier: S. 158-160, mit Abbildungen. 
Darstellungsstrategien von Atem, Atmung, Atemluft ausrichten. Ist die Gewährleistung der Sauerstoffversorgung auch im Film eine Grundvoraussetzung der Raumfahrt, die in der Gestaltung von Raumschiffen und Raumanzügen unterschwellig stets präsent ist, so wird die Atmung der Protagonistinnen und Protagonisten erst im Spektrum zwischen leichtem Stress und tödlicher Katastrophe dramaturgisch relevant und erfährt erst hier volle audio-visuelle Darstellbarkeit.

In The Martian (USA 2015) wird der Raumanzug von Matt Damon als Mark Watney im Chaos eines plötzlich auf dem Mars heraufziehenden Sturms beschädigt. Druck- und Sauerstoffverlust sind visuell nicht direkt, sondern nur im Effekt innerhalb der Spielhandlung erfahrbar. Sie werden den Zuschauerinnen und Zuschauern durch Watneys Körpersprache und verzerrte Miene, wiederum durch einen Fishbowl Helmet erkennbar, zu sehen und dem Publikum als Zuhörern und Zuhörerinnen durch Watneys keuchende Atmung im Kontrast zur ruhigen weiblichen Stimme des überwachenden Computers zu hören gegeben: „Oxygen level critical!“" Bei einem weiteren Unfall springt das Visier von Watneys Helm. Visuell effektvoller veranschaulicht sich der Sauerstoffverlust nun im Netz der Risse, durch das unsichtbar, aber unmittelbar assoziativ die Luft entweicht und das mit Klebeband hektisch notrepariert wird. $\mathrm{Zu}$ hören sind das Keuchen des bedrohten Mannes und wiederum die weibliche Computerstimme, die sanft die Kompromittierung der lebenserhaltenden Systeme verkündet. Harmloser gestaltet sich ein Ausflug mit dem Rover. Die Druckkabine gestattet den Verzicht auf einen Helm, die Instrumente zeigen angenehme 21,01\% Sauerstoff in der Atemluft, aber Watney hat, um Energie zu sparen, die Heizung ausgeschaltet. Die unangenehmen $-16,1^{\circ} \mathrm{C}$ lassen dem Astronauten die ausgeatmete Luft als Wölkchen vor dem Mund stehen. ${ }^{8}$

Die Darstellbarkeit von Atmung in Gefahrensituationen vertraut innerhalb der audio-visuellen, also lediglich bisensuellen Möglichkeiten des Mediums häufig genug auf assoziative Substitution und Komplementarität von Elementen der Diegese. In Mimi Leders Deep Impact (USA 1998) ziehen sich Astronauten auf einem Kometen hastig zurück, als die Sonne aufgeht und Eis geysirartig zu verdampfen beginnt. Die Sicht auf die Beine der gehemmt-stolpernd Fliehenden ist unterlegt mit einem universalen Geräusch stoßartiger Atmung, das sich nach einem

7 Ridley Scott, The Martian, DVD, Fox 2016, TC 00:10:20, zitiert nach der gesprochenen Version im Film.

8 Ebd., um TC 00:35:00. 
Schnitt zum realistischen Raumklang innerhalb des Helmes eines der Astronauten wandelt. Der Blick der Kamera gibt sich suggestiv als point of view-Einstellung, als subjektiver Blick des Protagonisten durch sein Helmvisier. Tatsächlich ist es aber nicht die Perspektive des Protagonisten, sondern ein Kamerastandpunkt schräg hinter seinem Kopf, weder subjektiver Blick noch Helmkamera oder ähnlich intradiegetisch Realistisches. Der auditive Eindruck behinderter Atmung verstärkt sich komplementär durch das visuell vermittelte Gefühl der Klaustrophobie, das als des Astronauten eigene Wahrnehmung suggeriert wird, während aber zugleich sein angespanntes Gesicht mit dem nach Luft schnappenden Mund gezeigt werden kann. Auf Atemwolken wird verzichtet; diesen Aspekt übernehmen substitutiv die Gaswolken des verdampfenden Eises außerhalb des Raumanzuges. ${ }^{9}$ Vergleichbare Strategien sind filmhistorisch Legion.

\section{Kreatur und Apparat}

Auch in der kinematographischen Raumfahrt verortet sich die Atmung der ins All Geschossenen zwischen Kreatürlichkeit und Technizismus, zwischen natürlichem Vorgang des lebenden Organismus - vorzüglich im Ausnahmefall des Keuchens, Nach-Luft-Schnappens - und der apparativen Gewährleistung und Überwachung des menschlichen Sauerstoffverbrauchs. Ridley Scotts The Martian, eine Mischung aus Robinsonade und Saving Private Ryan, nutzt dieses Spannungsfeld durchgehend zur dramaturgischen Strukturierung der Handlung. Der auf dem Mars versehentlich zurückgelassene Held und sein Umfeld werden immer wieder im Blick von Überwachungskameras gezeigt, Suitcams, Dashcams, Logcams, die die Filmbilder mit Anzeigen zu den Parametern der technischen Einrichtungen überlagern. Entspannt diktiert Robinson Watney im Wohntrakt der Logcam seinen Tagebucheintrag, und die Angaben im Display unterstreichen die stressfreie Situation. Der gemessene Luftdruck beträgt 12,48 psi, der Sauerstoffgehalt der Luft $20,79 \%$, die Raumtemperatur $21,15^{\circ} \mathrm{C} .{ }^{10}$ Unverändert präsentiert sich das Rahmenwerk der Display-Angaben, die diesmal zu Watneys Suitcam gehören, während eines katastrophalen Druckverlusts der Wohnkuppel - im panisch verrissenen Blick der subjektiven Kamera nun Tohuwabohu, in den Datenanzeigen jedoch dieselbe unbeteiligte

9 Mimi Leder, Deep Impact, DVD, Dream Works/ Paramount Pictures/Amblin Entertainment 1998, TC 00:51:20-00:51:32.

10 Die Angaben nach The Martian 2016 (wie Anm. 7), TC 00:15:20. 
Objektivität des Messbaren. Die Parameter in Watneys Raumanzug liegen im Normbereich, die der Umgebung sind lebensbedrohlich: Druck 0,11 psi, Sauerstoff 0,14\%, Temperatur $-61,9^{\circ} \mathrm{C}$ (Abb. 2).

Dramaturgische Valenzen entfaltet die Atmung zwischen Organismus und Maschine in den Science Fiction-Standardfilmszenen des Erwachens aus dem Hyper- oder Kryoschlaf, aus hibernation und Stasis, in die die Crew während sehr langer Raumflüge versetzt wurde. In Morton Tyldums Passengers (USA 2016) weckt das System Chris Pratt als Jim Preston: Jim, schlafend“ im Schneewittchen-Sarg seines Hibernation Pod mit den Displays zu den technischen Daten - Injektion - ein Schock läuft durch den Körper - Jims Lunge füllt sich mit Luft, sein Brustkorb hebt sich, der Körper fällt zurück - erster tiefer Atemzug - die Sensoren werden entfernt - Jim schlägt die Augen auf. Dieser prototypische erste Atemzug markiert exakt den Moment, an dem die Atmung, Pars pro Toto für sämtliche Lebensfunktionen, aus dem Verantwortungsbereich der Maschine in den des sich selbst erhaltenden Organismus (zurück-)gegeben wird: „Passenger discharged“, verkündet das Display. ${ }^{11}$

Die Science Fiction-Filmgeschichte entwarf zahlreiche Varianten für diesen signifikanten Atemzug. Grob lässt sich vielleicht eine Linie ziehen, die von der Simulation sanften natürlichen Erwachens hin zur gewaltsamen Reanimation führt, eine Entwicklung, die mit dem Wandel der Filmraumfahrt vom technizistisch glatten und weißen Entdeckerpathos der 1960er Jahre hin zur Alltäglichkeit im used look der 1970er und zu den dystopischen Welten bis in die Gegenwart korrespondiert. Je weiter die Filmraumfahrt voranschritt, desto brachialer wurde die Technologie des Hyperschlafs.

In Alien von 1979 beginnt das Erwachen in weißem Ambiente und milchig-lichter Atmosphäre als leichtes Räkeln. Die Unterwäsche der Schlafenden in ihren weich gepolsterten Pods erinnert entfernt an Windeln, das Aufrichten assoziativ an einen Wechsel in den Lotossitz und das erste Luftholen an Atemtechniken in meditativer Trance (Abb. 3). ${ }^{12}$ Die Szene erhält eine New Age-Aura, die noch dezidiert an Kubricks 2001: A Space Odyssee (Großbritannien/USA 1968) anknüpft, um sofort im Anschluss mit der handfest-schmuddeligen Stimmung des ersten Mannschaftsfrühstücks kontrastiert zu werden. Die Nostromo ist ein

11 Vgl. Morton Tyldum, Passengers, DVD, Sony Pictures

Home Entertainment 2017, TC 00:03:50-00:05:22.

12 Vgl. Alien 2012 (wie Anm. 5), TC ca. 00:04:05-00:04:30. 
Handelsschiff, Alien ein Markstein in der filmhistorischen Entwicklung hin zu düsteren Science Fiction-Welten und einer Szenographie des used (industrial) look.

James Camerons Fortsetzung Aliens (USA 1986) zeigt denn auch das Erwachen von Marines, darunter auch einigen weiblichen, im Stile eines Army Boot Camps inklusive Drill Sergeant und Dog Tags zwischen den Elektroden auf wohltrainierten Oberkörpern. ${ }^{13}$ Hier können die ersten Atemzüge auch mit Husten und Würgen wie nach durchzechter Nacht einhergehen als machistische Variante der heftigen Wiederaneignung der eigenen Lebensfunktionen. Weitere Spielarten wären die Steigerung der Säuglingskonnotate aus Alien I zur deutlichen Geburtsanalogie des schreienden schleimbedeckten Neugeborenen, das Erbrechen oder auch die Kombination existentieller Urängste vor dem Ersticken und Ertrinken, wenn die Gase in den Atmungsorganen während des Hyperschlafs durch Flüssigkeiten ersetzt wurden.

Wohl kaum etwas semantisiert aber die Übereignung des Atemvorgangs von der Maschine zum Menschen so prägnant wie ein kleines Detail aus Camerons Alien II. Als Georg Seeßlen befand, ,[d]ie Zukunft war im Kino schon immer ziemlich rauchfrei: In Raumschiffen und Superfabriken wird nicht geraucht ", ${ }^{14}$ entfiel ihm offensichtlich kurzzeitig die Erinnerung an die düsteren Science Fiction-Welten der 1970 er und 80er Jahre, zu denen quarzende Raubeine wie selbstverständlich dazugehörten. Geraucht wurde schon in Alien I, um der Luftaufbereitung des Raumschiffs etwas zu tun zu geben, das nicht zu den unabdingbaren Aufgaben der lebenserhaltenden Systeme gehörte. In Alien II nun steckt sich Al Matthews als Sergeant Apone filmhistorische sieben bzw. handlungsimmanente 57 Jahre später beim Aufwachen aus dem Hyperschlaf sofort sein Zigarillo zwischen die Lippen (Abb. 4). Wenn auch noch unangezündet, so geht sein erster Atemzug damit suggestiv durch den Glimmstängel, geht die Atemluft gemeinsam mit dem Tabakrauch in die Lungen und wird gemeinsam mit ihm wieder ausgeatmet. Der Vorgang bereichert auf erster Ebene den testosteronsatten Charakter der Mise en Scène, nicht zuletzt durch die hier bereits als hollywoodtypisch selbstironisches Zitat ausgestellte Konnotation erektiler Allzeitbereitschaft. Zugleich aber veranschaulicht das Detail, wie die Atmung aus der Rationalität der Maschine, die res-

13 Vgl. James Cameron, Aliens, DVD, Twentieth Century Fox Home Entertainment 2010, TC 00:20:50-00:22:12.
14 Georg Seeßlen, „Eine letzte Zigarette. Und schadet's der Gesundheit, es war auch eine Kultur: Fragmente einer Sprache des Rauchens, die kommenden Generationen fremd sein wird“, in: Deutsches Filminstitut (Hg.): Thank you for Smoking. Die Zigarette im Film, München 2014, S. 208-213, hier: S. 211. 

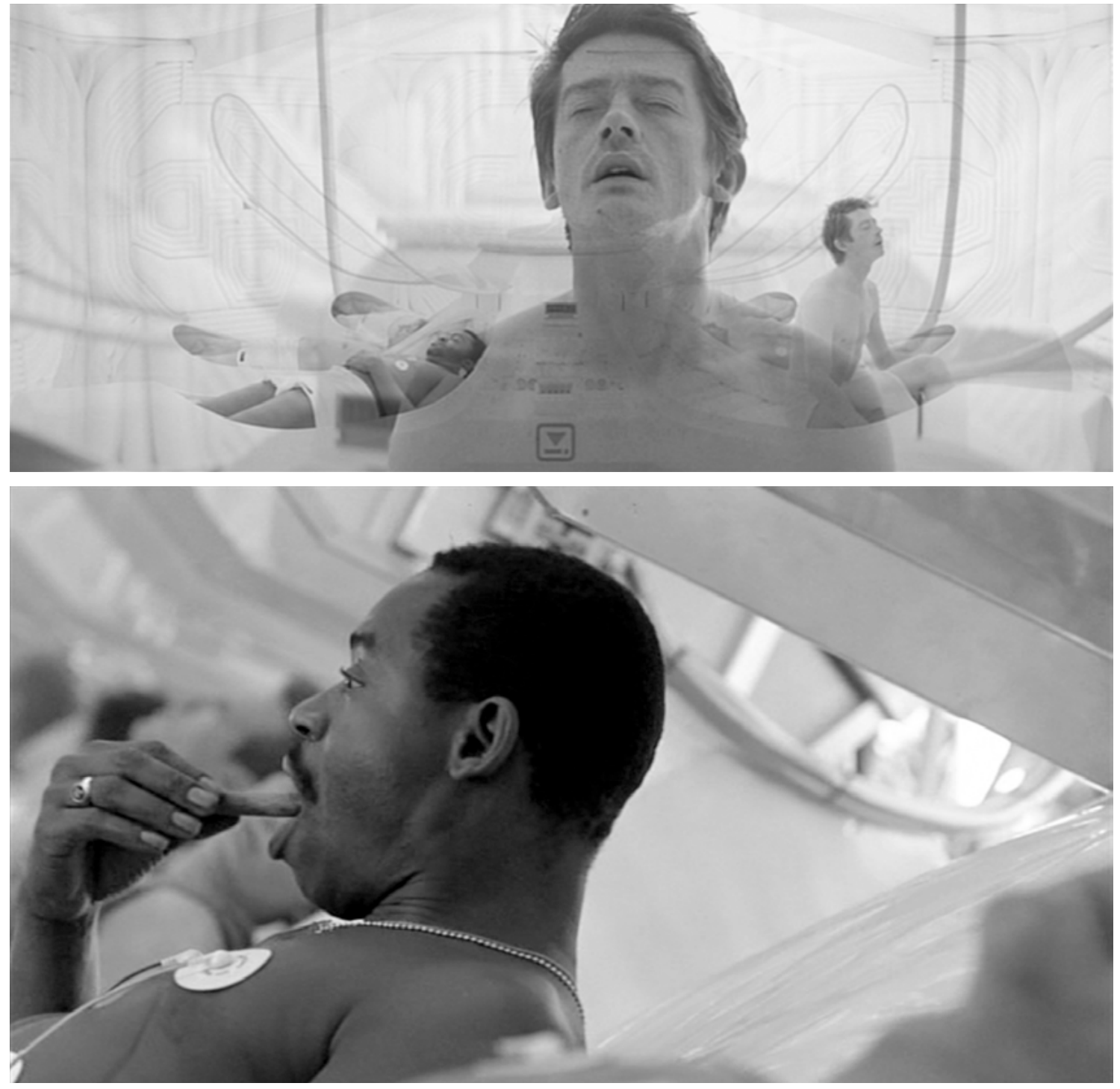
sourcenschonend die in der Stasis verminderten Körperfunktionen medizinisch zweckdienlich überwacht, entlassen und nicht nur wieder einem Organismus überantwortet wird, sondern einem Menschen. Die Atmung ist nicht nur Funktion ahistorischer Kreatürlichkeit, sondern sofort wieder in kulturelle Kontexte und Praktiken eingebettet, hier in die Kulturtechnik des Rauchens, ins Unvernünftige, nicht Zweckdienliche, in soziales Surplus. ${ }^{15}$

In Korrespondenz mit Entwicklungen der realen Raumfahrttechnik, vor allem aber einhergehend mit der Krise des Fortschrittsoptimismus, wandelten sich im Kino die silbern stromlinienförmigen Raketen der 1950er und die glatten weißen Raumkreuzer der 1960er à la Enterprise in den 1970er Jahren zu komplexen dunklen Gebilden. Die verästelt ausdifferenzierten Raumschiffe überziehen sich nun im Innern wie Äußern mit den Netzen von Versorgungsleitungen und technischen Systemen, deren Funktion undurchschaubar bleibt. Das Raumschiff wird zu einem Organismus mit unheimlichem Eigenleben, in dessen inneren Organen im Ernstfall das Verderben lauert - oder die Rettung zu finden ist. Wie bei Astronaut und Astronautin ist es wiederum die Atmung, die stellvertretend für alle Lebensfunktionen steht. Der Einstieg in den verborgenen Organismus des Schiffes erfolgt fast immer über die Schächte der Luftversorgung, nicht über Kabelschächte, Müllschlucker o.ä., und wiederum in der Not treten die existentiellen, aber sonst selbstverständlichen und unbemerkten Atemwege ins Bewusstsein und werden zum alternativen Erschließungssystem als letzter Ressource.

In Alien, 1979, beginnt der Einstieg in die Lüftungssysteme in einem hellen Interieur (sozusagen, wie beim skizzierten Erwachen aus dem Hyperschlaf, noch auf den ,hellen` Raumschiffen des vorangegangenen filmhistorischen Jahrzehnts). Die Kamera fährt zurück ins Dunkle; im Durchgang sind bereits Druckbehälter zu sehen; Sigourney Weaver als Ripley schließt die helle Schleusentür. Schnitte: Ripley und ihr Kollege mit Scheinwerfer ausgestattet in einem düsteren Technikraum. Ein Lamellenverschluss öffnet mit scharrendem Geräusch das Lüftungssystem, zugleich an die Verschlusstechnik einer Kamera erinnernd wie an organische Ventile. ${ }^{16}$ Dahinter beginnen

15 Der Überfluss des Rauchens jenseits der Effizienz dann auch herausgestellt in ebd., S. 212.

16 Vgl. Alien 2012 (wie Anm. 5), TC ca. 01:05:45-01:07:00. 
die Luftschächte als heart of darkness (in dem bekanntlich ein übelgelauntes Alien lauert).

Beim Showdown macht sich, nicht nur in diesem Film, das lebenserhaltende Element aus den verborgenen Systemen selbstständig und ist nicht mehr zu kontrollieren. Druckluft dringt auf der Nostromo, im Selbstzerstörungsmodus, aus allen Öffnungen und trägt als Theaternebel zum allgemeinen Chaos bei. Ripley, ohne Raumanzug, keucht. Sie rettet sich in ein Shuttle, aber das Alien ist auch hier mit an Bord. Damit wird die Situation invertiert: Ripley steigt in einen Raumanzug; ihr keuchender Atem lässt das Visier des schützenden Helms von innen beschlagen. Sie öffnet nun selbst die Druckluftvorräte und die Ausstiegsluke. Die gewaltige Dekompression und der Verlust sämtlicher Sauerstoffvorräte bringen die Rettung: das Alien wird in den Weltraum geschleudert. ${ }^{17}$ In The Martian wird sich Watney, den ein Loch im Helm beinahe umgebracht hätte, am Ende selbst ein Loch in den Raumanzug schneiden, um durch den Rückstoß der entweichenden Atemluft doch noch die Rettungsmannschaft im Orbit des Mars zu erreichen. ${ }^{18}$

\section{Der Tank und der Garten, Tankwart und Gärtnerin}

Speicherung und Verlust der mitgeführten Sauerstoffvorräte sind zentrale Narrateme im Science Fiction-Film. In der älteren Tradition ist es der Tank mit seinem quantifizierbaren Volumen, der sich mit technizistischen Phantasien von Machbarkeit und Berechenbarkeit verbindet. Seine Beschädigung im dramatischen Moment erfolgt durch gewaltsame Penetrationen der Hülle, durch Meteoriten- und anderen Beschuss. Die Notwendigkeit, das Ausströmen des Lebensstoffes mit allen Mitteln zu stoppen, legt den Vergleich mit dem gewaltsam durch Schuss oder Stich penetrierten Körper des männlichen Helden nahe. Gelingt es nicht oder nur unzureichend, die Wunde zu versorgen oder die Hülle eines Tanks zu flicken, müssen nach Blut- respektive Sauerstoffverlust die Ressourcen neu berechnet werden: wer und wie viele kommen mit dem Verfügbaren noch bis wohin, reitend ins nächste Fort oder raumfahrend zur Erde?

Bereits Thea von Harbous Roman Frau im Mond sowie ihr Drehbuch und die Verfilmung des regieführenden Gatten Fritz Lang von 1929 konstruierten solche Analogien durch eine enge Verknüpfung von

17 Die letzte Phase des Showdowns in ebd., TC ca. 01:47:00-01:48:30.

18 Vgl. The Martian 2016 (wie Anm. 7), TC ab 02:02:00. 
Ereignissen der Handlung. Bei einer lunaren Schießerei wird der Schurke Walt (im Film: Walter) Turner tödlich getroffen; sterbend verhöhnt er den unverletzten Kontrahenten:

„,Mein Schuß... hat nicht... Ihnen gegolten, Helius', stammelte er. ,Er sollte... viel tiefer treffen... und hat auch getroffen...'

,Was meinen Sie damit, Turner...

,Sie werden es sehen..." [...]

,Ihr werdet nicht... auf die Erde... zurückkehren...' sagte der Sterbende. ,Keiner, keiner von... euch..." 19

Nur zu bald erkennt Wolf Helius, was der Schurke gemeint hatte:

„,Turner sagte, sein Schuß habe nicht mir gegolten; er sollte viel tiefer treffen - er hat getroffen... Er hat die gekoppelten Ventile der Sauerstoffapparate zerstört, und weit mehr als die Hälfte unseres Vorrats an Sauerstoff, den wir für den Rückflug zur Erde brauchten, ist ausgeströmt..." 20

„Und was bedeutet das praktisch?“,

fragt genderkonform die einzige Frau im Expeditionsteam, um sofort vom Manne belehrt zu werden:

„,Nichts', antwortete Hans Windegger mit einer fieberhaften Geschwindigkeit, ,nichts als ein wunderbares Rechenexempel! Ein Kind kann es begreifen! Sieh her! Wenn wir zur Rückfahrt neunzig Stunden rechnen, so brauchen wir vier Erwachsene und das Kind rund 230 Millionen Kubikzentimeter Sauerstoff. 250 Millionen hatten wir im Vorrat. Davon ist über die Hälfte ausgeströmt, nämlich 136 Millionen. Es bleibt uns also ein Rest von 114 Millionen Kubikzentimeter das heißt: für neunzig Stunden Rückfahrt zur Erde Atemstoff für zwei und einen halben Menschen, also für zwei Erwachsene und ein Kind... ist das klar?

,Vollkommen“, sagte das Mädchen still.“ 21

Und so wird es Zeit fürs Strohhalmziehen, für männliche Opferbereitschaft, in der sich der wahre Held erweist, und fürs trickreich noch größere weibliche Opfer, das die Liebe gebietet. Friede Velten bleibt mit Helius, im Kino immerhin verkörpert von Ufa-Frauenschwarm Willy 
Fritsch, auf dem Mond zurück. Fritz Langs Stummfilm kam mit weniger Worten aus als der Roman, eröffnete aber einen bis heute andauernden kinematographischen Reigen von zu berechnenden Ressourcenverlusten an Atemluft. Bereits sieben Jahre später wird in Vasili Zhuravlovs Kosmicheskiy reys (Kosmische Reise, UdSSR 1936) der nächste Sauerstofftank bei der Landung des Raumschiffs Josef Stalin auf dem Mond beschädigt.

In jüngerer Zeit tauchen in Auseinandersetzung mit Überlegungen innerhalb der realen bemannten Raumfahrt, zu Geoengineering bzw. Terraforming und im Kontext wachsenden ökologischen Bewusstseins ${ }^{22}$ auch im Film verstärkt Konzeptionen auf, in denen an die Stelle von Speicherung und Verbrauch Produktion und Reproduktion von Sauerstoff treten. In Danny Boyles Sunshine (Großbritannien 2007) fliegt die Icarus II zur Sonne, um deren schwächer werdendes Fusionsfeuer mittels einer immensen Sprengladung wieder anzufachen und die Erde vor dem Kältetod zu bewahren. Die Luftversorgung an Bord gewährleistet ein Sauerstoffgarten. Er wird uns vorgestellt, indem der Blick der Kamera unter sanfter Musik über die filigrane Spindel der Icarus im Schatten ihres gigantischen Sonnenschutzschildes schweift bis zu einem rechteckigen Fenster, hinter dem Grünes sichtbar wird. Der Schnitt ins Innere bringt das Geräusch von Regen und fokussiert Details wie Ventilatoren und Algenbewuchs, der die sauberen weißen Flächen erobert, bevor eine Totale mit leichtem Kameraschwenk den blaugrünen Hauptraum des Gewächshauses erschließt: Wasser, aufragende Pflanzen, an den Seiten Reihen kreisrunder Öffnungen mit hellgrüner beleuchteter Vegetation, vor der sich die Rotorblätter von Ventilatoren drehen. Offensichtlich wird hier der photosynthetisch erzeugte Sauerstoff bewegt. Schnitt, Großaufnahme: Möhrenernte. ${ }^{23}$

Mit dem Paradigmenwechsel zur natürlichen Re-Generation des lebenserhaltenden Sauerstoffs wird, kulturpolitisch geradezu zwangsläufig, aus dem männlichen Ingenieur des Ressourcen-Managements die ökologisch umsichtige weibliche Gärtnerin Corazon. Die Sorge um die Atemluft wird zu einem der,weichen“ Themen, mit denen Frauen traditionell bei ihren ersten Auftritten im Science Fiction-Film betraut wurden: Friede Velten, 1929 zwar als Astronomiestudentin die erste

22 Zum Kontext des Terraforming sei hier nur auf zwei einflussreiche Klassiker verwiesen: Carl Sagan, „The Planet Venus“, in: Science 133 (3456), 1961, S. 849-858 und Martyn J. Fogg, Terraforming: Engineering Planetary Environments, Warrendale 1995; zum Stand der Überlegungen und aktuellen Projekten zu Pflanzen im All siehe die Informationsseite der NASA: https://www.nasa.gov/ audience/foreducators/spacelife/topics/plants/index. html (letzter Zugriff: 5.6.2020).
23 Vgl. Danny Boyle, Sunshine, DVD, Twentieth Century Fox Home Entertainment 2007, TC ca. 00:06:10-00:06:40. 

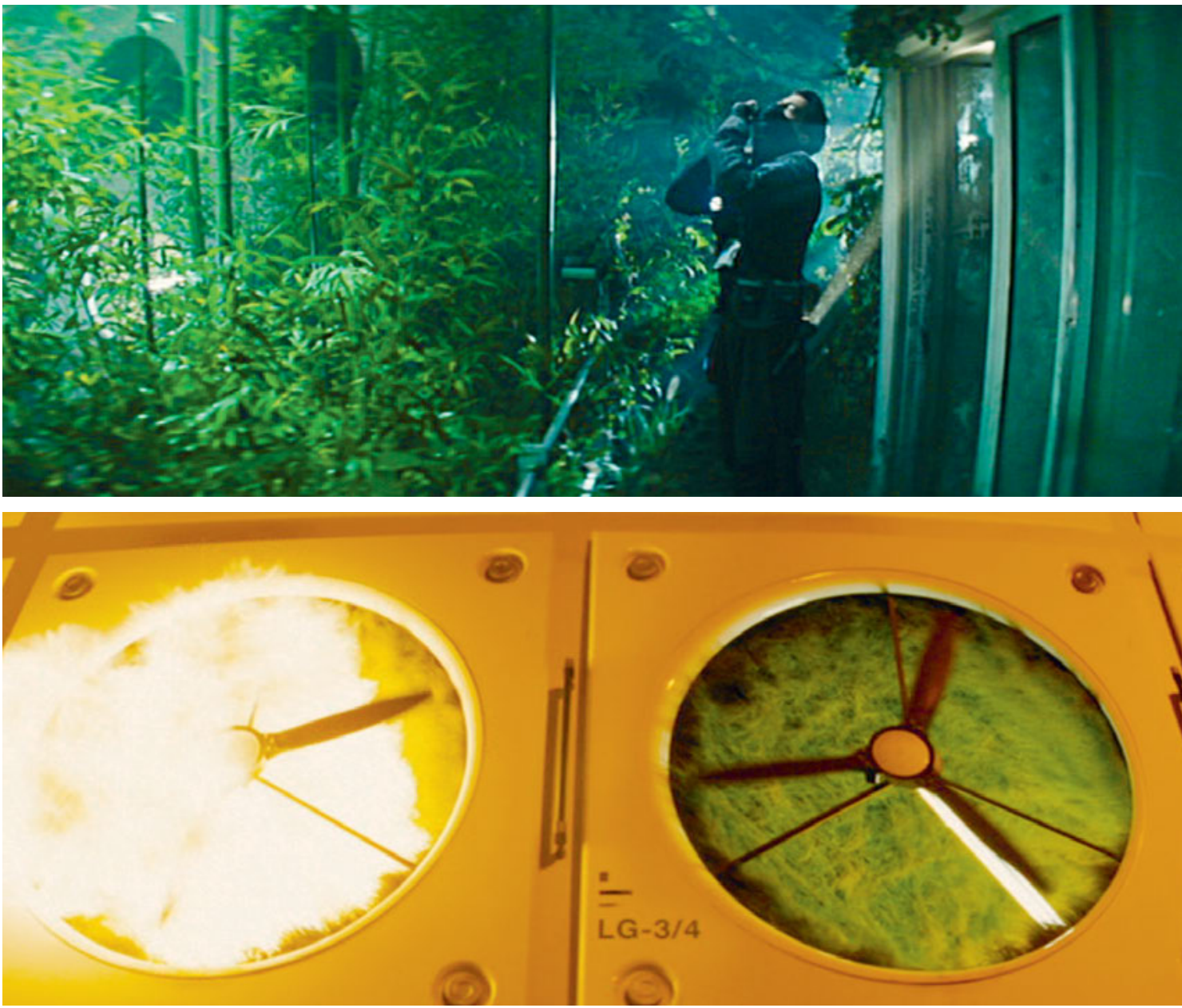

Abb. 5: Verwilderter Sauerstoffgarten auf der Icarus I, Sunshine, 2007, Screenshot
Abb. 6: Zerstörung des Sauerstoffgartens, grün zu gelb, Sunshine, 2007, Screenshot 
Frau im Mond, doch als Verlobte des Ingenieurs Windegger vor allem auch ,die Frau an seiner Seite; Sumiko Ogimura als Bordärztin im Schweigenden Stern von 1960, die seit dem Atombombenabwurf auf Hiroshima keine Kinder bekommen kann; Lieutenant Uhura in Star Trek seit 1966 als qua Geschlecht kommunikativ kompetente Kommunikationsoffizierin. 1979 wird Ripley in Alien diese Gendernormativität kräftig aufmischen.

Als weibliche Domäne geht der Sauerstoffgarten auf der Icarus nicht ins Freie, Weite und Offene, ins Betätigungsfeld der eminenten männlichen Gartenkünstler Le Nôtre, Capability Brown, Fürst Pückler von der Frühen Neuzeit bis ins ausgehende 19. Jahrhundert, sondern bleibt ein Gewächshaus, in dem zarte Pflänzchen zu beschirmen und zu behüten sind. ${ }^{24}$ Auffällig sind die Konnotate des Asiatischen und die Aura der Wellness-Oase im Fernost-Chic. Die Ventilatorenöffnungen erinnern an die kreisrunden Mondtore der klassischen chinesischen Gartenkunst; der Regen plätschert leis'; es gibt Bambusartiges; alles ist frisch, rein und beruhigend. Kaum zufällig wird die Sauerstoff-Gärtnerin Corazon (Herz!) mit Michelle Yeoh von einer malaysischen Schauspielerin chinesischer Abstammung dargestellt. Seine deeskalierend psychotherapeutische Effizienz beweist der Sauerstoffgarten etwa, wenn er zum Ort der zwischenzeitlichen Versöhnung der stets körperlich aggressiv aufeinander losgehenden männlichen Crewmitglieder Capa und Mace wird; von Corazon ist ein belustigter Stoßseufzer zu hören..$^{25}$

Wie stark dieser Garten ethno-gegendert ist, beweist sein Gegenstück auf der Icarus I, dem verschollenen Raumschiff der gescheiterten Vorgängermission, das die Icarus II im Schatten des Merkur findet. Obwohl der dortige Garten exakt dasselbe macht wie Corazons Gewächshaus, nämlich Sauerstoff produzieren, entpuppt er sich als $a$ man's world. Immer noch voll funktionsfähig, haben ihn sieben Jahre ungehinderten Wachstums zum Dschungel werden lassen. Astronaut Harvey reißt sich das Tuch vom Gesicht, das vor dem Staub schützt, der

24 Das genderhistorische Feld am Beispiel Deutschlands wird etwa abgesteckt bei Annette Dorgerloh, „Weibliche und männliche Gartenwelten“, in: Stefan Schweizer/ Sascha Winter (Hg.), Gartenkunst in Deutschland. Von der Frühen Neuzeit bis zur Gegenwart. Geschichte Themen - Perspektiven, Regensburg 2012, S. 219-232. Hingewiesen sei jedoch auch auf jüngere filmische Korrekturversuche wie Alan Rickmans A Little Chaos (Die Gärtnerin von Versailles, Großbritannien 2014).
25 Vgl. Sunshine 2007 (wie Anm. 23), TC ca. 00:11:40. Bemerkenswerterweise startete Michelle Yeoh ihre Karriere aber als Star in ostasiatischen Action- und Martial ArtsFilmen. In der UK-Produktion Sunshine darf sie hingegen noch sterbend ein neues Pflänzchen als Baby beschirmen, während der frühe männliche Space-Gärtner Freeman Lowell in Douglas Trumbulls Silent Running (USA 1972) die von ihm gepflegten Biosphären auch zum Kampfplatz macht. Watney in The Martian ist Botaniker und ebenfalls Gärtner, sein Ziel ist allerdings der Anbau von Nahrungsmitteln. Über die Pflanzungen reflektiert Watney im Bezugsfeld von Inbesitznahme und Kolonisation; vgl. The Martian 2016 (wie Anm. 7), TC um 00:57:30. Die Produktion von Sauerstoff wird im Film kaum thematisiert; ein oxygenator ist vorhanden. 
sonst alles auf der unheimlich verlassenen Icarus I bedeckt, nimmt einen tiefen befreienden Atemzug und schwingt sich über das Geländer ins Unterholz (Abb. 5). Technische Einrichtungen des Gartens, die Mondtor-artigen Ventilatorenöffnungen, die es auch hier geben muss, kommen kaum ins Bild. Der männliche Held dringt, seine Angst bezwingend, in den Urwald vor, in dem vielleicht das Monster wartet, das die erste Mission scheitern ließ. ${ }^{26}$

Auf der Icarus II aber fängt das weiblich gepflegte Garten-Gegenstück Feuer bei einem riskanten Manöver mit dem Einstellwinkel des Sonnenschildes. ${ }^{27}$ Die auch hier entspannt weibliche Stimme des Bordcomputers verkündet, das Feuer werde sechs Stunden im abgeschotteten Garten brennen, das Risiko einer Beschädigung der Schiffshülle betrage $60 \%$, das von Kollateralschäden lebenserhaltener Systeme 75\%. Mace gibt den Befehl, den bereits produzierten Sauerstoff ausströmen zu lassen, damit sich das Feuer in einem Flammensturm selbst ausblase - Cassie, Astronautin: „We'1l lose the whole garden!“; Mace, Astronaut: „We already lost it.“ Im Inferno des Brandes färben sich die Filmbilder des einst blaugrünen Gartens in das Gelborange der Sonnenglut, von dem vor allem die männlichen Besatzungsmitglieder im gesamten Film wie besessen sind (Abb. 6). Der Garten mit seiner Fähigkeit, den Atemsauerstoff zu regenerieren, wird vollständig vernichtet; die Gärtnerin Corazon ist am Boden zerstört. Während der suggestiv männliche Dschungel-Garten auf der Icarus I seine lebensspendende Potenz gerade in der auflösenden Verwilderung beweist, ist es auf der Icarus II das sorgsam ,geerntete' Gartenprodukt Sauerstoff selbst, das zum Todesstoff wird. Die Inversion als Memento intrinsischer Vergeblichkeit?

Denn auch auf diesem Filmraumschiff beginnen nun wieder das Ressourcenrechnen und das Streichholzziehen um die verbleibende Atemluft. Der Bordcomputer entdeckt aufgrund zu hohen Sauerstoffverbrauchs einen gefährlichen blinden Passagier. ${ }^{28}$ Capa und Mace prügeln sich wieder und liegen nach Luft ringend am Boden; die Frauen geben den genervten Rat, sie sollen sich wieder einkriegen - man müsse Sauerstoff sparen.

26 Vgl. ebd., TC ca. 00:50:50-00:52:00.

27 Vgl. ebd., TC ab 00:34:05; Zitate nach der gesprochenen Version im Film.
28 Der aufschlussreiche Dialog zwischen Capa und dem in der weiblichen Computerstimme personifizierten Raumschiff vollständig auch unter https://en.wikiquote.org/ wiki/Sunshine_(2007_film) (letzter Zugriff: 6.5.2020). 